\title{
On the oscillations of spatial vibration-isolating system of mining machines under the action of impact loads
}

\author{
Mykola Lysytsia ${ }^{1, *}$ \\ ${ }^{1}$ Institute of Geotechnical Mechanics named by N. Poljakov of National Academy of Sciences of \\ Ukraine, 49005, Dnipro, Simferopolska Str., 2a, Ukraine
}

\begin{abstract}
Theoretical and experimental results concerning interaction between lumpy loads and loading sections of a belt conveyer have been represented. The rational parameters of the loading section of the conveyor belt are determined from the point of view of reducing the amplitude of oscillations after the interaction. The regularities of oscillation amplitude of a loading section after interaction with a single lump have been identified.
\end{abstract}

\section{Introduction}

Since conveyer transport is high-productive, it is the integral part of schemes of cyclic and continuous flow process technology as well as continuous flow process technology in mining. Loading of lumpy product and its conveyance is the restricting factor to apply belt conveyers efficiently.

Currently, loading sections of belt conveyers with the use of steel ropes to support rollers and carrying rollers are very popular.

Effect of lumpy product on carrying rollers of loading section of a conveyer is characterized by pressure of dice load as well as impact of lumps with certain time interval. After the lump impact, carrying rollers of the section oscillate relative to balance position. When following lump is falling, relative velocity of interaction of the bodies (i.e. loadcarrying roller) may either increase or decrease to compare with the initial impact due to carrying roller oscillations. The fact results in the increased dynamic loads both on the belt and the carrying rollers. To decrease the loads, it is necessary for time of suspension system of carrying rollers balancing to be less than a period during which lumps pass. In this context, it is necessary to know displacement mode of carrying rollers, and determine effect of structure of the section and its parameters on its displacement mode in time.

Consideration of the problem is of prime importance while designing supporting loading elements taking into consideration a flow of the material being loaded.

The paper analyzed oscillations of the two basic types of loading sections - with conveying ropes, and nonrigid carrying rollers designed by Institute of Geotechnical Mechanics named by N. Poljakov of National Academy of Sciences of Ukraine (Fig. 1).

${ }^{*}$ Corresponding author: vita.igtm@gmail.com 


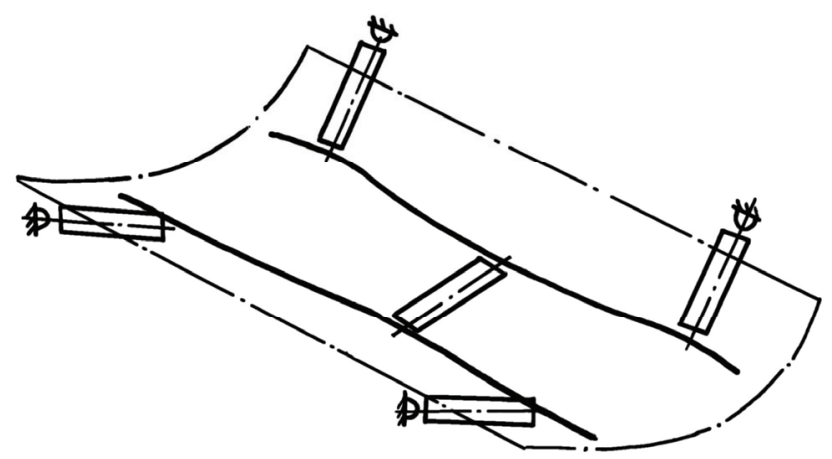

Fig. 1. Section of nonrigid carrying rollers.

\section{Theoretical study}

While forming oscillation equation of nonrigid carrying roller section, the following has been hypothesized: movement value of central roller cannot depend upon load point along the section length, and longitudinal movements of the rollers are more than by an order of magnitude lesser than vertical ones; and when nonrigid carrying rollers interact with a lump, rope tension varies if only suspension achieves its maximum displacement. Further oscillations of the section take place in terms of practically constant rope tension. That makes it possible to consider a section of nonrigid carrying rollers as a constant density string on an elastic foundation; in this context, at a first approximation, damping of the system is not considered.

Figure 2, a demonstrates analytical model where $U$ is string deviation from its balance; $L$ is string length; $T$ and $\rho$ are string tension and linear density respectively; and $C_{0}$ is rigidity coefficient of the elastic foundation. Figure 2,b shows original form of the string bend where $b$ is distance between central rollers and $h$ is the string bend. Original form of the string bend depends upon design features of the section of nonrigid carrying rollers; moreover, experimental data confirm the fact.

a)

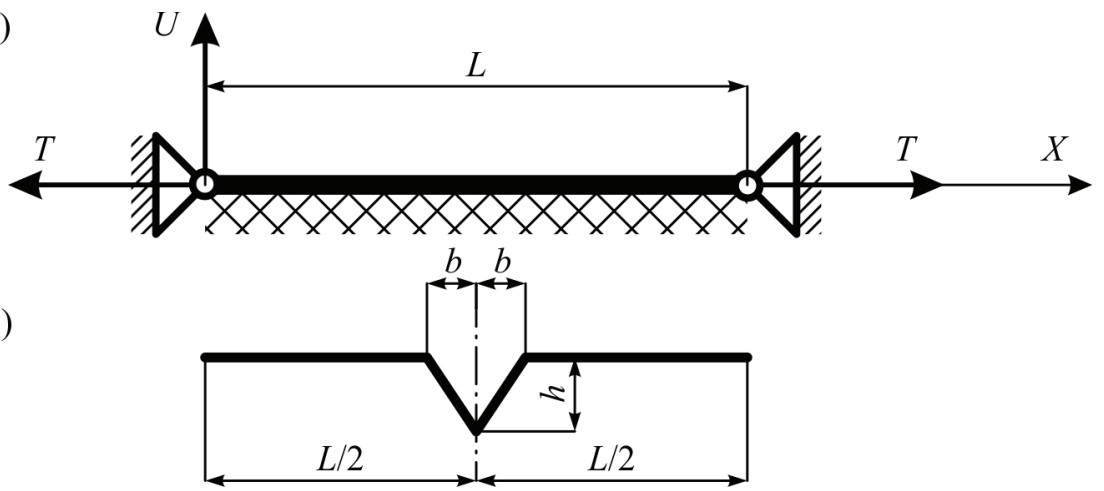

Fig. 2. Analytical model of a section of nonrigid carrying rollers.

Using the principles of d'Alembert [1] for the string section we obtain the equations of oscillations:

$$
\frac{\partial^{2} U}{\partial x^{2}}=\beta^{2} \frac{\partial^{2} U}{\partial t^{2}}+k^{2} U
$$


where $\beta^{2}=\frac{\rho}{T} ; k^{2}=\frac{C_{0}}{T}$,

in terms of following initial conditions:

$$
\begin{aligned}
& U(x, 0)= \begin{cases}0, & 0<x<\frac{L}{2}-b ; \\
-\frac{h}{b} x+\frac{h}{b}\left(\frac{L}{2}-b\right), & \frac{l}{2}-b<x<\frac{L}{2} ; \\
\frac{h}{b} x-\frac{h}{b}\left(\frac{L}{2}+b\right), & \frac{L}{2}<x<\frac{L}{2}+b ; \\
0, & \frac{L}{2}+b<x<L ;\end{cases} \\
& \frac{\partial U(x, 0)}{\partial t}=0
\end{aligned}
$$

and boundary conditions:

$$
\begin{aligned}
& U(0, t)=0 \\
& U(L, t)=0
\end{aligned}
$$

The solution of equation (1) by the Fourier method is presented in general form:

$$
U(x, t)=X(x) T(t)
$$

where

$$
\begin{gathered}
X(x)=A \cos (\sqrt{\lambda} x)+B \sin (\sqrt{\lambda} x) \\
T(t)=C_{n} \cos \left(\sqrt{\frac{k^{2}}{\beta^{2}}+\frac{\lambda}{\beta^{2}}} \cdot t\right)+D_{n} \sin \left(\sqrt{\frac{k^{2}}{\beta^{2}}+\frac{\lambda}{\beta^{2}}} \cdot t\right) \\
\lambda=\left(\frac{\pi n}{L}\right)^{2}, \quad(n=1,2,3 \ldots),
\end{gathered}
$$

$A, B, C_{n}, D_{n}-$ arbitrary constants.

Insert expression (5) into equation (4). While using conditions (2), and (3) we obtain:

$$
U(x, t)=\sin \left(\frac{n \pi}{L} x\right)\left\{C_{n} \cos \left[\sqrt{\left(\frac{k}{\beta}\right)^{2}+\left(\frac{n \pi}{L \beta}\right)^{2}} \cdot t\right]+D_{n} \sin \left[\sqrt{\left(\frac{k}{\beta}\right)^{2}+\left(\frac{n \pi}{L \beta}\right)^{2}} \cdot t\right]\right\} .
$$

Relying upon $\frac{\partial U(x, 0)}{\partial t}=0$ condition, we identify that $D_{n}=0$.

Then,

$$
U(x, t)=\sum_{n=1}^{\infty} C_{n} \cos \left[\sqrt{\left(\frac{k}{\beta}\right)^{2}+\left(\frac{n \pi}{L \beta}\right)^{2}} \cdot t\right] \sin \left(\frac{n \pi}{L} x\right)
$$


In terms of equation (9), constant $C_{n}$ is determined using the expression:

$$
C_{n}=\frac{2}{L} \int_{0}^{L} f(x) \sin \left(\frac{n \pi}{L} x\right) d x
$$

where $f(x)$ is a function corresponding to initial conditions (2).

It follows from initial conditions (2) that:

$$
f(x)= \begin{cases}0, & 0 \leq x<\frac{L}{2}-b \\ -\frac{h}{b} x+\frac{h}{b}\left(\frac{L}{2}-b\right), & \frac{L}{2}-b \leq x<\frac{L}{2} \\ \frac{h}{b} x-\frac{h}{b}\left(\frac{L}{2}+b\right), & \frac{L}{2} \leq x<\frac{L}{2}+b \\ 0, & \frac{L}{2}+b \leq x<L .\end{cases}
$$

Inserting expressions (8) into (7), we obtain

$$
C_{n}=\frac{8 h L}{b^{2} \pi^{2} n^{2}} \sin \left(\frac{n \pi}{2}\right) \sin ^{2}\left(\frac{n \pi}{2} \frac{b}{L}\right)
$$

Taking into consideration that $\beta^{2}=\rho / T$, and inserting (9) into (6), we obtain definitely:

$$
U(x, t)=-\frac{8 h L}{b \pi^{2}} \sum_{n=1}^{\infty} \frac{1}{n^{2}} \sin ^{2}\left(\frac{n \pi}{2} \frac{b}{L}\right) \sin \frac{n \pi}{2} \cos \left[\sqrt{\frac{C_{0}}{\rho}+\frac{T}{\rho L^{2}}(n \pi)^{2}} \cdot t\right] \sin \left(\frac{n \pi}{L} x\right) .
$$

According to [2], rigidity coefficient of elastic foundation is determined using the expression

$$
C_{0}=\frac{p}{f b}
$$

where $p$ is a load acting on the roller; and $f$ is displacement of the roller under the load action.

Consider oscillations of loading section with conveying ropes. In this context, boundary conditions remain previous ones, and initial conditions are:

$$
\begin{aligned}
& U(x, t)= \begin{cases}-\frac{2 h}{L} x, & 0<x<\frac{L}{2} ; \\
\frac{2 h}{L}(x-L), & \frac{L}{2}<x<L ;\end{cases} \\
& \frac{\partial U(x, 0)}{\partial t}=0 .
\end{aligned}
$$

If initial boundaries (11), and boundary ones (3) are taken into consideration, then solution of equation (1) is 


$$
U(x, t)=-\frac{8 h}{\pi^{2}} \sum_{n=1}^{\infty} \frac{1}{n^{2}} \sin \left(\frac{n \pi}{2}\right) \cos \left[\sqrt{\frac{C_{0}}{\rho}+\frac{T}{\rho L^{2}}(n \pi)^{2}} \cdot t\right] \sin \left(\frac{n \pi}{L} x\right) .
$$

Hence, expressions have been developed to determine displacements of loading section points for nonrigid carrying rollers (10), and sections with conveying ropes (12).

We will analyze the solutions obtained.

Study the effect of loading section length, and initial deviation nature on its oscillation mode within a point of maximum bend, i.e. if $x=L / 2$, and $U(x, 0)=-h$.

1. Suppose that $L=2 b$, i.e. initial deviation corresponds to (11) conditions. Then, expression (10) is

$$
U\left(\frac{L}{2}, t\right)=-\frac{16 h}{\pi^{2}} \sum_{n=1}^{\infty} \frac{1}{n^{2}} \sin ^{2}(n \pi) \cos \left[\sqrt{\frac{C_{0}}{\rho}+\frac{T}{\rho L^{2}}(n \pi)^{2}} \cdot t\right] \sin ^{2}\left(\frac{n \pi}{2}\right) .
$$

If $n$ values are even ones, then expression (13) is equal to 0 . If $n$ values are odd ones, then $\sin ^{2} \frac{n \pi}{4}=\frac{1}{2}$. Hence, expression (13) is as follows

$$
U\left(\frac{L}{2}, t\right)=-\frac{8 h}{\pi^{2}} \sum_{n=1}^{\infty} \frac{1}{n^{2}} \sin ^{2}\left(\frac{n \pi}{2}\right) \cos \left[\sqrt{\frac{C_{0}}{\rho}+\frac{T}{\rho L^{2}}(n \pi)^{2}} \cdot t\right] .
$$

The generated dependence coincides competely with expression (12) for section with conveying ropes.

In accordance with (12), string points with coordinate $x=L / 2$ oscillate harmonically with following frequency

$$
\omega=\sqrt{\frac{C_{0}}{\rho}+\frac{T}{\rho L^{2}}\left(n \pi^{2}\right)}
$$

and amplitude being equal to $\pm 8 h / \pi^{2}$.

While setting $C_{0}=0$ in (12), we arrive at the known expression of oscillations of finitelength string with fixed ends [1].

2. Determine displacement if $L \rightarrow \infty$. While applying L'Hospital rule, we identify that

$$
U\left(\frac{L}{2}, t\right)=\lim _{L \rightarrow \infty} \frac{\left[\sum_{n=1}^{\infty} \frac{1}{n^{2}} \sin ^{2}\left(\frac{n \pi}{2} \frac{b}{L}\right)\right]^{11}}{\left(\frac{b \pi^{2}}{8 h L}\right)^{11}}=0
$$

i.e. in terms of considerable $L$, oscillations of sections decay within a point of maximum bend.

Calculations on formula (10) have been performed to determine effect of the section parameters on its oscillations. The section parameters varied as follows: distance between rollers $b$ was 0.8 and $1.0 \mathrm{~m}$; rope tension $T$ was $6.0 ; 8.0$ and $10.0 \mathrm{kN}$; section length $L$ was $5 ; 10 ; 15$ and $20 \mathrm{~m}$; linear density $\rho-50 ; 80 ; 110 \mathrm{~kg} / \mathrm{m}$; rigidness of nonrigid carrying rollers $C$ was $60.0 ; 90 ; 120$ and $150 \mathrm{kN} / \mathrm{m}^{2}$ and initial deviation was $h=0.09 \mathrm{~m}$. 
The number of series terms was determined with $10 \%$ calculation accuracy and amounted $11 ; 21 ; 31$ and 43 respectively for such section lengths as $5 ; 10 ; 15$ and $20 \mathrm{~m}$. For $1 \%$ calculation accuracy, 129, 257 and 385 series terms $(L=5 ; 10 ; 15 \mathrm{~m})$ required respectively. However, comparison of the obtained results has shown that the number of series terms effects calcualtion accuracy of initial ratio $h$ at $t=0$ time moment. If $t>0$, then displacement calculation divergence is up to $1.5 \%$.

The calculations results helped construct graphs of vertical dispacements of the section in time if its parameters vary.

Figure 3, a, demonstrates graphs of lateral oscillations of the sections in terms of its varied length as well as rigidness (Fig. 3, b) of elastic foundation. The graphs explain that in the course of time, the section oscillates with constant amplitude decreasing along with the increase in the section length. To compare with the initial disturbance, the amlitude decrease of subsequent oscillations is connected with the fact that at $t=0$ time moment, deviation from the balance concerns limited section area only. Then, the whole its length is involved in the oscillations. Analysis of curves in Figure 3,b shows that the increased rigidness of elastic foundation results in the increase of subsequent oscillations (curve 2). The increased amplitude can be explained by the increased potential energy of the section area with the initial deviation.

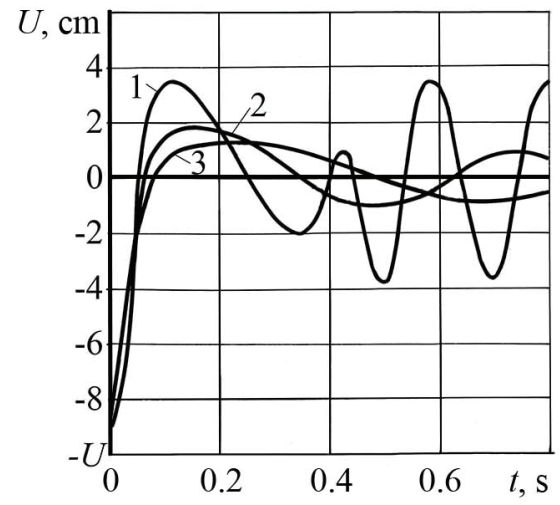

$1-L=5 \mathrm{~m} ; 2-L=10 \mathrm{~m} ; 3-L=20 \mathrm{~m}$

a) in terms of the varied section length

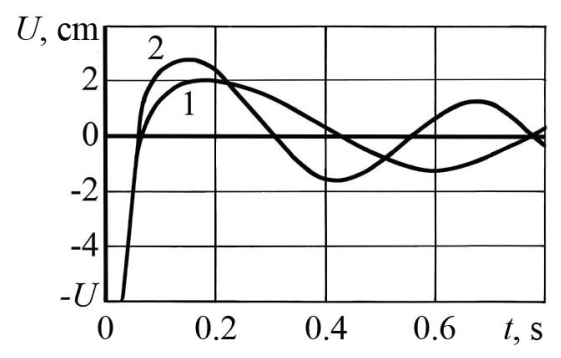

$$
1-60 \mathrm{kN} / \mathrm{m}^{2} ; 2-120 \mathrm{kN} / \mathrm{m}^{2}
$$

b) in terms of the varied rigidness of the elastic foundation

Fig. 3. Graphs of lateral oscillations.

Analysis of the calcualtion results has shown:

- changes in line load has minor effect on the amplitude of subsequent oscillations;

- increase in rope tension decreases amplitude of subsequent oscillations; and

$-1.0 \mathrm{~m}$ down to $0.8 \mathrm{~m}$ decrease in distance between rollers results in $25-30 \%$ decrease of subsequent section oscillations.

The research has made it possible to determine the effect of the loading section design and parameters on the mechanism of roller displacement reduction in the context of vertical oscillations of the loading section after its interaction with falling material. To compare with initial displacement, the mechanism intended to decrease amplitude of roller oscillation is as follows: during the first time moment, falling load localized bend within a small distance of the section length; subsequently, the energy, accumulated by the area, is consumed by longitudinal oscillations along the section length. Further, point along the section length oscillate. Amplitute of their oscillations is 8-10 times less to compare with the initial deviation amplitude resulted from the load impact. Consideration of frictional losses within the system will result in the damped oscillations while complicating the 
solution significantly. However, neither qualitative nor quantitative analysis may involve consideration of the losses.

It has been determined that 6-8 times decrease in amplitude of vertical oscillations of rollers during 0.4-0.5 $\mathrm{s}$ is provided owing to following section parameters: length should not be less than $10 \mathrm{~m}$; rope tension should be $8-10 \mathrm{kN}$, rigidness should be $100-120 \mathrm{kN}$, and distance between rollers should be $0.7-0.8 \mathrm{~m}$.

\section{Experiments}

The experiments were carried out by means of a belt conveyor set-up with $80 \mathrm{~m}$ length, $0^{\circ}$ inclination angle, and $800 \mathrm{~mm}$ belt width. Granite was loaded. Its maximum lump size was 400-800 mm; drop height was $0.5 \mathrm{~m}$ and $1.0 \mathrm{~m}$; nonrigid carrying rollers were used within loading area.

Objective of the experiments is to substantiate analytical model, and to make qualitative evaluation of the results of theoretical studies as for interaction between load and nonrigid carrying rollers.

Figure 4 demonstrates a scheme of arrangement of sensors within a section of nonrigid carrying rollers. Hereinafter, following symbols are applied:

- $D_{1}, D_{3}$, and $D_{4}$ are sensors of vertical displacements of central rollers;

$-D_{2}$ is a sensor of vertical displacements of central roller interacting with the falling load;

- $P_{1}$, and $P_{2}, C_{1}$, and $C_{2}$ are sensors of angular displacements of side rollers;

$-K_{1}$, and $K_{2}$ rope tension sensors.

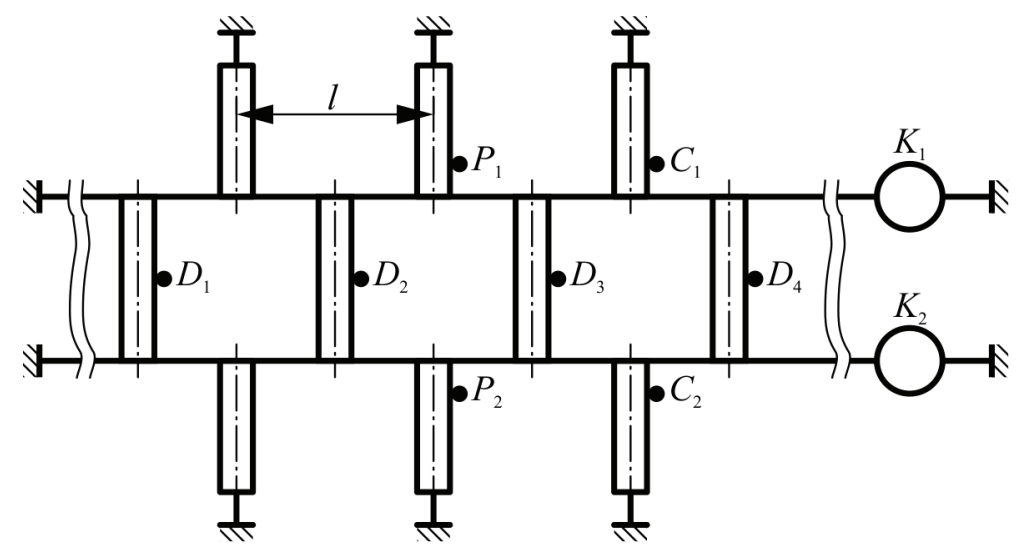

Fig. 4. Scheme of arrangement of sensors.

Values of displacement of rollers as well as their oscillation mode after impact with a lump load were determined if variations concerned: loading height $(0.5 \mathrm{~m} ; 0.6 \mathrm{~m} ; 0.8 \mathrm{~m}$ and $1.0 \mathrm{~m})$; falling lump weight (16 kg and $29 \mathrm{~kg})$; preliminary rope tension $(2.0 \mathrm{kN} ; 3.0 \mathrm{kN}$; and $4.0 \mathrm{kN}$ and distance between rollers $(1 \mathrm{~m})$.

Figure 5 explains interaction of $16 \mathrm{~kg}$ lump when rope tension is $4.0 \mathrm{kN}$.

Analysis of the obtained data shows that after impact, a roller performs rapidly damping oscillations during 0.4-0.5 s. Amplitude value of oscillations, following the interaction, are 6-10 times less to compare with maximum displacement during impact.

Movement of other central rollers (sensors $D_{1}$, and $D_{4}$ ) starts when a roller, experiencing the impact (sensor $D_{2}$ ), achieves maximum displacement. Hence, weigth of one roller takes place in the impact; loading section bends in terms of length being equal to a twofold pitch of arrangement of rollers. After the interaction, roller oscillations take place in the context of almost constant rope tension. Preliminary rope tension increase results in 
oscillation amplitude of the roller after interaction.

Availability of a belts is not very important for maximum roller displacements (difference is up to $10 \%$ ) having minor effect on the section oscillation mode.

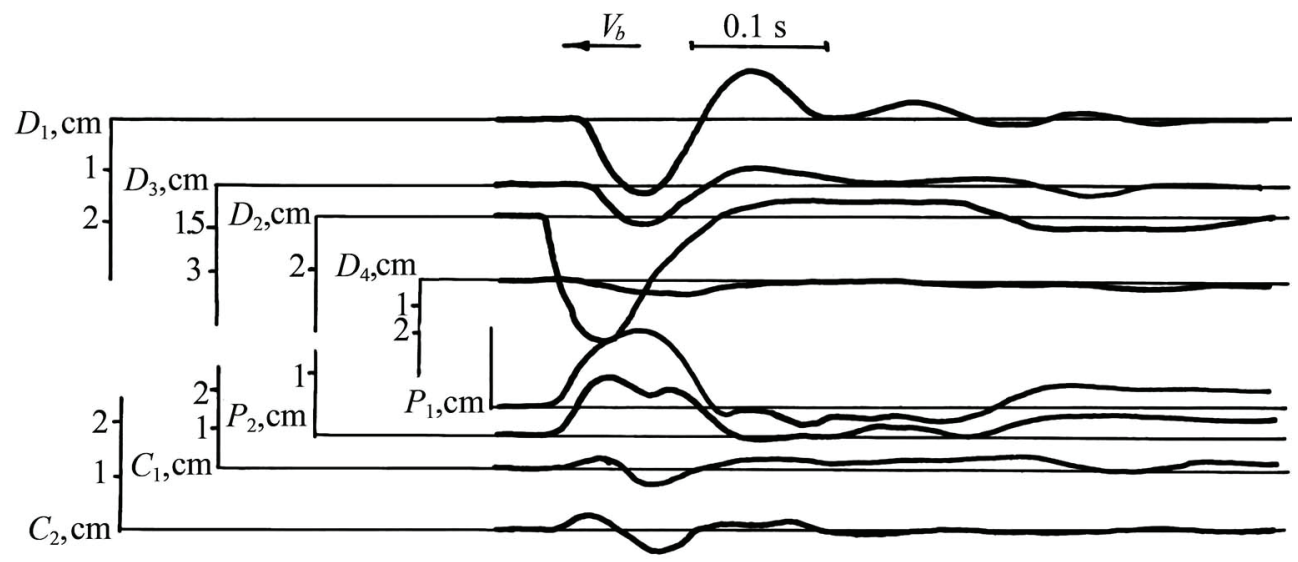

Fig. 5. Oscillorgams of interaction between a lump and nonrigid carrying rollers.

\section{Conclusions}

1. The experiments have confirmed adequacy of the assumptions originated during the analytical model substantation.

2. The results of theoretical studies, concerning the effect of loading section parmeters on the mode of vertical oscillations, and on their amplitute after interaction with a single lump, have been supported.

3. The regularities of oscillation amplitude of a loading section after interaction with a single lump have been identified.

\section{References}

1. Aramanovich, I.G., Levin, V.I. (1969). Uravneniya matematicheskoy fiziki. Moskva: Nauka

2. Timoshenko, S.P. (1946). Soprotivleniye materialov. Moskva: Gostekhizdat 